\title{
Adaptive Control for Nonlinear Systems with Time-Varying Control Gain
}

\author{
Alejandro Rincon ${ }^{1}$ and Fabiola Angulo \\ ${ }^{1}$ Programa de Ingeniería Ambiental, Facultad de Ingeniería y Arquitectura, Universidad Católica de Manizales, Carrena 23 No. 60-30, \\ Manizales 170002, Colombia \\ ${ }^{2}$ Departamento de Ingeniería Eléctrica, Facultad de Ingeniería y Arquitectura, Universidad Nacional de Colombia, Sede Manizales, \\ Electrónica y Computación, Percepción y Control Inteligente, Bloque Q, Campus La Nubia, Manizales 170003, Colombia
}

Correspondence should be addressed to Fabiola Angulo, fangulog@unal.edu.co

Received 21 November 2011; Accepted 11 April 2012

Academic Editor: Chengyu Cao

Copyright (C) 2012 A. Rincon and F. Angulo. This is an open access article distributed under the Creative Commons Attribution License, which permits unrestricted use, distribution, and reproduction in any medium, provided the original work is properly cited.

We propose a scheme for nonlinear plants with time-varying control gain and time-varying plant coefficients, on the basis of a plant model consisting of a Brunovsky-type model with polynomials as approximators. We develop an adaptive robust control scheme for this plant, under the following assumptions: (i) the plant terms involve time-varying but bounded coefficients, being its upper bound unknown; (ii) the control gain is unknown, not necessarily bounded, and only its signum is known. To achieve robustness, we use a combination of robustifying control inputs and dead zone-type update laws. We apply this methodology to the speed control of a permanent magnet synchronous motor (PMSM), and we achieve proper tracking results.

\section{Introduction}

Nonlinear behavior is difficult to model accurately, rendering controller design cumbersome. An approach to handle this is to use linear models, which have local validity in the state space, that is, different values of the parameter set would be required for each region in the state space. A second approach is to use a plant model in either the Brunovsky form defined in [1], or the parametric-pure feedback form defined in [2], using the so-called "function approximation techniques", such as locally weighted statistical learning [3], fuzzy sets [4], Fourier series [5], orthonormal functions, [6] or neural networks [7]. In the last case, state and time dependent terms, known as "basis functions," are used to represent the nonlinear and time-varying behaviour. Some identification or learning methods are used to perform the approximation, resulting in a negligible modelling error if there are a sufficient number of basis functions [3]. In some cases, a Brunovsky form model of the plant is used, assuming that there is full-state measurement and that the basis functions are multiplied by unknown constants. Then, a sliding surface model reference adaptive control (SSMRAC) is devised, handling the residual approximation error by means of auxiliary robustifying inputs [7].

Common drawbacks of the above-mentioned schemes are

(i) the convergence of the tracking error to some small residual set is not ensured in a strict sense and depends on the value of the approximation error [7];

(ii) upper or lower bounds of the plant coefficients are required to be known [8];

(iii) discontinuous auxiliary inputs are used, which may lead to chattering [5];

(iv) high enough gains are used, which require excessive values [6].

Due to environment changes, the coefficients of the plant model may experience time-varying but bounded behavior $[9,10]$. Both, the control gain and other coefficients may exhibit this behavior. The time-varying behavior of coefficients different from the control gain may be handled by 
means of robustness techniques. See for instance the robust MRAC scheme in [10], whose drawback is that projectionor $\sigma$-type update laws are used, and hence upper bounds of the plant coefficients are required to be known, in order to achieve the convergence of the tracking error to some residual set of user-defined size. The time-varying control gain may be handled by means of robustness techniques [11] or Nussbaum gain technique [12], a projection-type update law is used, such that a lower bound of the control gain is required to be known. In [11], a $\sigma$ type update law is used, such that bounds of the plant coefficients are required to be known. In summary, the main drawback of using robustness techniques to handle varying control gain is that upper or lower bounds of some plant coefficients are required to be known. On the other hand, the Nussbaum gain technique relaxes this requirement. The main drawback is that the upper bound of the transient behavior of the state $S$ is significantly altered with respect to that of the disturbancefree case: the value of this bound depends on time integrals of the terms that comprise the Nussbaum function. Another drawback is that the control gain is usually required to be upper-bounded by some constant.

The scheme of [13] achieves adequate robustness properties. Mainly, upper bounds of the plant coefficients are not required to be known. The essential element of the technique is to define the quadratic forms related to the sliding surface $\delta$ in terms of a dead zone function of $\delta$ rather than in terms of $\&$. This scheme has the following benefits.

(Ri) The tracking error converges to a residual set whose size is of the user's choice;

(Rii) known upper bounds of the plant coefficients are not required, such that high enough gains are not used;

(Riii) all the closed-loop signals are bounded (parameter drifting is avoided);

(Riv) auxiliary control signals are not discontinuous in terms of both the tracking error and the sliding surface, hence input chattering is avoided;

(Rv) upper bounds of time-varying but bounded coefficients are not required to be known.

The disadvantages of [13] are (i) the design is only developed for systems with hysteresis nonlinearities in the actuator; (ii) the time-varying control gain is tackled by means of the Nussbaum gain method, which entails higher complexity of the Lyapunov analysis; (iii) the control gain is assumed to be time-varying but bounded.

In contrast to the approaches of $[3,4,14-16]$, we propose that an adequate regression model for highly nonlinear systems may be obtained from a Brunovsky type model, inserting polynomials to approximate the nonlinear behavior, and considering also: (i) time-varying but bounded behavior of some plant coefficients; (ii) unknown control gain, timevarying, and not necessarily bounded. We develop a robust adaptive scheme for this plant, achieving benefits ( $\mathrm{Ri}$ ) to $(\mathrm{Rv})$. If we compare the robust technique developed here with the technique developed in [13], the main differences, which are also contributions, are

(Rvi) we consider unknown time-varying control gain, not necessarily bounded, not restricted to actuator nonlinearities;

(Rvii) we consider time-varying but bounded behavior of some plant terms;

(Rviii) we tackle the control gain by means of robustness techniques, which gives a simpler design in comparison with the Nussbaum technique.

This paper is organized as follows. The outline of the scheme is given in Section 2. The function approximation is discussed in Section 3. The plant regression model and the statement of the problem are established in Section 4. The control and update laws are derived in Section 5. The control algorithm and its stability are presented in Section 6. An application of the scheme to a PMSM is given in Section 7. Finally, the conclusions are presented in Section 8.

\section{Outline of the Scheme}

We propose the use of polynomials to approximate the nonlinear behavior, taking into account the fact that polynomials are universal approximators for continuous functions, according to [17]. We consider a Brunovsky-form plant model, into which we introduce the polynomial terms.

We devise a robust adaptive controller for this plant, achieving benefits ( $\mathrm{Ri}$ ) to (Rix) mentioned in the introduction. We use the SSMRAC method stated in [18], as the basic framework for designing the control and update laws. To handle the effect of modelling error and timevarying behavior of plant terms, we use a robust continuous control input, whose magnitude is adjusted, and a dead zonetype update law. The resulting controller has the following features: (i) the magnitude of the control input is adjusted to cope with the unknown upper bounds of the timevarying coefficients; (ii) the control input is proportional to some continuous function of the sliding surface $\delta$, so that chattering is avoided; (iii) an inactivation is introduced in all the update laws, which occurs when the sliding surface 8 reaches a target region. If we compare this with projectiontype update laws, it has the advantage of not requiring the upper bounds of the plant parameters.

For the stability analysis, we use a truncated version of the quadratic form related to the sliding surface, denoted by $\overline{V_{s}}$. The validity of this technique, including the conditions of the Lyapunov function, is stated in [19]. It is worth noticing that the standard conditions of the Lyapunov theory for time-variable systems, shown in [18] or [20], are not satisfied because of the truncation. We prove the asymptotic convergence of the tracking error in a rigorous manner by means of Barbalat's Lemma. To that end, we redefine the expression of $\dot{V}$ as an inequality in terms of $\overline{V_{s}}$. 


\section{Function Approximation Based on Polynomials}

According to [17, page 116], a continuous real-valued function $\bar{f}(x)$, where $x \in D, D \subset R^{n}$, being $D$ a compact set, may be approximated by a polynomial function $\bar{g}$ in the interval $x \in D$, with some finite error, being $\bar{g}$ defined as

$$
\bar{g}\left(x, \theta_{o}\right)=\sum_{k_{1}, k_{2}, \ldots, k_{n}} \theta_{o[j]} x_{1}^{k_{1}} x_{2}^{k_{2}} \cdots x_{n}^{k_{n}}: \quad \theta_{o} \in \Omega, \Omega \subset R^{p},
$$

where $\Omega$ is a convex set and $\theta_{o[j]}$ means the $j$ th element of the vector $\theta_{0}$. Thus, $\bar{f}(x)$ can be expressed in terms of $\bar{g}$ as follows (cf. [17] page 122):

$$
\begin{gathered}
\bar{f}(x)=\bar{g}\left(x, \theta_{a}\right)+\epsilon_{o}(x), \quad\left|\epsilon_{o}(x)\right|<\epsilon, \\
\theta_{a}=\left\{\theta_{o} \in \Omega: \theta_{o}=\arg \min _{\theta_{o}}\left(\sup _{x \in D}\left|\bar{f}(x)-\bar{g}\left(x, \theta_{o}\right)\right|\right)\right\},
\end{gathered}
$$

where $\epsilon$ is a positive constant, and it is known as approximation error, $\theta_{a}$ is an ideal parameter set and $\bar{g}\left(x, \theta_{a}\right)$ is an ideal representation of $\bar{f}(x)$. The polynomial can be linearly parameterized with respect to its coefficients:

$$
\bar{f}(x)=\phi^{\top} \theta_{a}+\epsilon_{o}(x),
$$

where $\phi$ contains polynomial terms, whereas $\theta_{a}$ contains constants. In this work, $\bar{f}$ represents the nonlinear part of the dynamical equation. We will handle the unknown constant vector $\theta_{a}$ by means of adjustment rules and $\epsilon_{o}$ by means of robust techniques.

\section{Plant Model and Problem Statement}

We assume that the dynamical nonlinear system can be represented by a Brunovsky type model, as defined in [1], with polynomial functions:

$$
\begin{gathered}
y^{(n)}=-c_{n-1} y^{(n-1)}-\cdots-c_{1} \dot{y}-c_{o} y+\bar{f}(x)+b u, \\
x=\left[y, \dot{y}, \ldots, y^{(n-1)}\right]^{\top},
\end{gathered}
$$

where $y(t) \in \mathbb{R}^{1}$ is the system output $u(t) \in \mathbb{R}^{1}$ is the input and the coefficients $c_{n-1}, \ldots$, and $c_{o}$ are unknown and time-varying but bounded. The relative degree $n$ may be established by means of previous step response analysis. Inserting the function (3) into (4) gives

$$
\begin{gathered}
y^{(n)}=b u+\phi^{\top} \theta_{a}+d, \\
d=\epsilon_{o}(x)-c_{n-1} y^{(n-1)}-\cdots-c_{1} \dot{y}-c_{o} y, \\
\left|c_{n-1}\right| \leq \mu_{n-1}, \ldots,\left|c_{1}\right| \leq \mu_{1}, \\
\left|c_{o}\right| \leq \mu_{n}, \quad\left|\epsilon_{o}(x)\right| \leq \mu_{n+1}=\epsilon .
\end{gathered}
$$

We make the following assumptions:

(Ai) the entries of $\theta_{a}$ and the terms $\mu_{1}, \ldots$, and $\mu_{n+1}$ are constant and unknown;
(Aii) the control gain $b$ varies with respect to the state $x$, or time, so that it satisfies the following:

(i) $0<b_{m} \leq|b| \leq f_{b}, \quad \forall t \geq t_{o}$,

(ii) $\operatorname{sign}(b)$ constant, $\quad \forall t \geq t_{o}$,

where the value of $\operatorname{sign}(b)$ is known, $b_{m}$ is an unknown positive constant, and $f_{b}$ is an unknown positive function of time or $x$. Notice that condition (8) implies that $b$ is always different from zero;

(Aiii) the entries of the vector $x$ are available for measurement;

(Aiv) the entries of the vector $\phi$ are known functions of $x$;

(Av) the value of the desired trajectory $y_{d}(t)$ and its derivatives $y_{d}^{(n-1)}, \ldots, \dot{y}_{d}$ is bounded.

The desired output $y_{d}$ is specified in terms of a bounded external command $r(t)$ as follows:

$$
y_{d}^{(n)}+a_{m, n-1} y_{d}^{(n-1)}+\cdots+a_{m, o} y_{d}=a_{m, o} r,
$$

where $a_{m, n-1}, \ldots, a_{m, o}$ are constant coefficients prespecified by the user, such that the polynomial $K(p)$ is Hurwitz with at least one real root, being $K(p)$ defined as $K(p)=p^{(n)}+$ $a_{m, n-1} p^{(n-1)}+\cdots+a_{m, o}$. The external reference signal $r(t)$ must be bounded. The objective of the MRAC design is to formulate a control law $u(t)$ such that the tracking error $e(t)=y(t)-y_{d}(t)$ asymptotically converges to the residual set $D_{e}$, defined as follows:

$$
D_{e}=\left\{e:|e| \leq C_{b e}\right\},
$$

where $C_{b e}$ is a user-defined bound.

\section{The Control and Update Laws}

Let $\delta$ the dynamics imposes over the tracking error given by

$$
\delta=(p+\lambda)^{n-1} e=p^{n-1} e+\lambda_{n-2} p^{n-2} e+\cdots+\lambda_{1} \dot{e}+\lambda_{o} e,
$$

where $\lambda$ is a positive constant chosen by the user. Having defined $\delta$, we establish the dynamic equation for $\&$ by differentiating with respect to time:

$$
\begin{gathered}
\dot{\delta}=p^{n} e+\lambda_{n-2} p^{n-1} e+\cdots+\lambda_{1} \ddot{e}+\lambda_{o} \dot{e}, \\
\dot{\delta}=y^{(n)}-y_{d}^{(n)}+\lambda_{n-2} p^{n-1} e+\cdots+\lambda_{1} \ddot{e}+\lambda_{o} \dot{e}, \\
\dot{\delta}=y^{(n)}+\varphi_{a}, \\
\varphi_{a}=-y_{d}^{(n)}+\lambda_{n-2} p^{n-1} e+\cdots+\lambda_{1} \ddot{e}+\lambda_{o} \dot{e} .
\end{gathered}
$$


We insert the expression for $y^{(n)}$ of (6) into (15):

$$
\dot{s}=b u+\theta_{a}^{\top} \phi+d+\varphi_{a} .
$$

Define

$$
\begin{gathered}
u^{*}=-\theta_{a}^{\top} \phi-\varphi_{a}-a_{m} \S=\varphi^{\top} \theta^{*}, \\
\theta^{*}=\left[\theta_{a}^{\top}, 1\right]^{\top}, \\
\varphi=\left[-\phi^{\top},-\varphi_{a}-a_{m} \S\right]^{\top},
\end{gathered}
$$

where $\varphi_{a}$ is defined in (16), $a_{m}$ is a positive constant. We express (17) in terms of $u^{*}$ as follows:

$$
\dot{s}=-a_{m} \delta+b u+d-u^{*}=-a_{m} \delta+b u+d-\varphi^{\top} \theta^{*} .
$$

Let

$$
\begin{gathered}
l=n+1, \\
c^{*}=\frac{2+l}{2 C_{b v s}}\left(\frac{1}{2 \sqrt{b_{m n}}}\right)^{2} \\
C_{b v s} \triangleq\left(\frac{1}{2}\right)\left(\lambda^{n-1} C_{b e}\right)^{2}, \\
f_{1}=|\dot{y}|, \ldots, f_{l-2}=\left|y^{(n-1)}\right|, \\
f_{l-1}=|y|, \quad f_{l}=1, \\
\mu_{1}=\max \left\{\left|c_{1}\right|\right\}, \ldots, \mu_{l-2}=\max \left\{\left|c_{n-1}\right|\right\}, \\
\mu_{l-1}=\max \left\{\left|c_{o}\right|\right\}, \quad \mu_{l}=\epsilon .
\end{gathered}
$$

Now, we multiply (20) by $\&$ and add and subtract the term $c^{*} S^{2}$ :

$$
s \dot{s}=-a_{m} s^{2}+b s u+s d-s \varphi^{\top} \theta^{*}+c^{*} S^{2}-c^{*} S^{2},
$$

where the terms $c^{*} \delta^{2}-c^{*} \delta^{2},-\varsigma \varphi^{\top} \theta^{*}$, and $\delta d$ can be expressed in terms of adjustment errors:

(i) $c^{*} \delta^{2}-c^{*} \delta^{2}=-c^{*} \delta^{2}-\tilde{c} \delta^{2}+\hat{c} \delta^{2}, \quad \tilde{c}=\hat{c}-c^{*}$;

(ii) $\quad-s \varphi^{\top} \theta^{*}=S \varphi^{\top} \tilde{\theta}-s \varphi^{\top} \hat{\theta}, \quad \tilde{\theta}=\hat{\theta}-\theta^{*}$;

(iii) $\quad s d \leq(-1) \tilde{c}_{1}|\&| f_{1}+\hat{c}_{1}|\&| f_{1}+\cdots+(-1) \tilde{c}_{l}|\&| f_{l}+\hat{c}_{l}|\&| f_{l}$,

$$
\tilde{c}_{1}=\widehat{c}_{1}-\mu_{1}, \ldots, \tilde{c}_{l}=\widehat{c}_{l}-\mu_{l}
$$

where $\hat{c}, \hat{\theta}, \widehat{c}_{1}, \ldots, \widehat{c}_{l}$ are adjusted parameters whose update laws will be defined later. Inserting the above properties into (25) gives

$$
\begin{aligned}
& \delta \dot{\delta} \leq-a_{m} \delta^{2}-\tilde{c} \delta^{2}+\delta \varphi^{\top} \tilde{\theta}+(-1) \tilde{c}_{1}|\delta| f_{1} \\
& +\cdots+(-1) \tilde{c}_{l}|\&| f_{l}+\hat{c} \delta^{2}-\diamond \varphi^{\top} \hat{\theta}+\hat{c}_{1}|\&| f_{1} \\
& +\cdots+\hat{c}_{l}|\delta| f_{l}+b s u-c^{*} \delta^{2} \text {. }
\end{aligned}
$$

If $b$ were constant, we would choose the control $u$ so as to cancel the terms involving updated parameters $\hat{c}, \hat{\theta}, \hat{c}_{1}, \ldots, \hat{c}_{l}$. Since $b$ is varying, we chose $u$ to attenuate the effect of adjusted parameters, being the remaining error rejected by $-c^{*} s^{2}$ :

$$
\begin{aligned}
u= & (-1) \operatorname{sgn}(b) \hat{c}^{2} \delta^{3}+(-1) \operatorname{sgn}(b)\left(\varphi^{\top} \hat{\theta}\right)^{2} \& \\
& +(-1) \operatorname{sgn}(b) \hat{c}_{1}^{2} f_{1}^{2} \&+\cdots+(-1) \operatorname{sgn}(b) \hat{c}_{l}^{2} f_{l}^{2} \&,
\end{aligned}
$$

where $\hat{c}, \hat{\theta}, \hat{c}_{1}, \ldots, \widehat{c_{l}}$ are adjusted parameters whose update laws will be defined later. Replacing the above control law into (20) and (30) gives

$$
\begin{aligned}
\dot{s}= & -a_{m} s-|b| \hat{c}^{2} s^{3}-|b|\left(\varphi^{\top} \hat{\theta}\right)^{2} s \\
& -|b| \hat{c}_{1}^{2} f_{1}^{2} s+\cdots-|b| \hat{c}_{l}^{2} f_{l}^{2} s+d-\varphi^{\top} \theta^{*}, \\
s \dot{s} \leq & -a_{m} s^{2}-\tilde{c} s^{2}+s \varphi^{\top} \tilde{\theta}+(-1) \tilde{c}_{1}|s| f_{1} \\
& +\cdots+(-1) \tilde{c}_{l}|s| f_{l}+\hat{c} s^{2}-s \varphi^{\top} \hat{\theta}+\hat{c}_{1}|s| f_{1} \\
& +\cdots+\hat{c}_{l}|\&| f_{l}-|b| \hat{c}^{2} s^{4}-|b|\left(\varphi^{\top} \hat{\theta}\right)^{2} s^{2}-|b| \hat{c}_{1}^{2} f_{1}^{2} s^{2} \\
& -\cdots-|b| \hat{c}_{l}^{2} f_{l}^{2} s^{2}-c^{*} s^{2},
\end{aligned}
$$

where the last terms of the above equation satisfy the following inequality (see the proof in Appendix A):

$$
\begin{aligned}
\hat{c} s^{2} & -s \varphi^{\top} \hat{\theta}+\hat{c}_{1}|\&| f_{1}+\cdots+\hat{c}_{l}|\&| f_{l}-|b| \hat{c}^{2} s^{4} \\
& -|b|\left(\varphi^{\top} \hat{\theta}\right)^{2} s^{2}-|b| \hat{c}_{1}^{2} f_{1}^{2} s^{2}+\cdots-|b| \hat{c}_{l}^{2} f_{l}^{2} s^{2} \\
& -c^{*} s^{2} \leq 0 \quad \text { if } s^{2} \geq 2 C_{b v s} .
\end{aligned}
$$

Equation (34) expresses the attenuation of the effect of the adjusted parameters. Substituting (34) into (33) gives

$$
\begin{gathered}
\delta \dot{\delta} \leq-a_{m} \delta^{2}-\tilde{c} \delta^{2}+\delta \varphi^{\top} \tilde{\theta}-\tilde{c}_{1}|\&| f_{1} \\
-\cdots-\tilde{c}_{l}|\&| f_{l} \quad \text { if } \delta^{2} \geq 2 C_{b v s}
\end{gathered}
$$

or equivalently,

$$
\begin{gathered}
s \dot{s} \leq-a_{m} s^{2}-\tilde{c} s^{2}+s \varphi^{\top} \tilde{\theta}-|s| \tilde{C}_{d}^{\top} f \quad \text { if } s^{2} \geq 2 C_{b v s} \\
\widetilde{C}_{d}=\left[\tilde{c}_{1}, \ldots, \tilde{c}_{l}\right]^{\top}, \quad f=\left[f_{1}, \ldots, f_{l}\right]^{\top}
\end{gathered}
$$

We choose the following update laws:

$$
\begin{gathered}
\dot{\hat{c}}= \begin{cases}\gamma_{c} \&^{2} & \text { if } \AA^{2} \geq 2 C_{b v s}, \\
0 & \text { otherwise, }\end{cases} \\
\dot{\hat{C}}_{d}= \begin{cases}\Gamma_{d} f|\&| & \text { if } \AA^{2} \geq 2 C_{b v s}, \\
0 & \text { otherwise, }\end{cases} \\
\dot{\hat{\theta}}= \begin{cases}-\Gamma \varphi \& & \text { if } 8^{2} \geq 2 C_{b v s}, \\
0 & \text { otherwise, }\end{cases}
\end{gathered}
$$

where $\&$ is defined in (12), $C_{b v s}$ in (23), whereas $\gamma_{c}$, and the diagonal entries of $\Gamma, \Gamma_{d}$ are positive constants chosen by the user, being $\Gamma$ and $\Gamma_{d}$ diagonal matrices. 


\section{The Control Algorithm}

Now we recall the equations corresponding to the controller and establish the tracking convergence theorem. The control law is given by (31); the update laws are given by (38); the terms $\varphi$ and $\delta$ are defined in (19) and (12); $\varphi_{a}$ is defined in (16); $C_{b v s}$ is defined in (23) and $f$ is defined in (37). The adjusted parameters $\hat{c}_{1}, \ldots, \hat{c}_{l}$, required to compute $u$, are the entries of the vector $\hat{C}_{d}$, that is, $\widehat{C}_{d}=\left[\hat{c}_{1}, \ldots, \hat{c}_{l}\right]^{\top}$.

6.1. Theorem: Boundedness and Tracking Convergence. If the controller designed in Section 5 is applied to the plant (6), then the tracking error $e(t)$ converges to $D_{e}$ asymptotically, and the closed-loop signals remain bounded.

Proof. Now we proceed to analyze the stability of the controlled system using the direct Lyapunov method and the Barbalat's Lemma. First, we establish the boundedness of the closed-loop signals on the basis of the time derivative of the Lyapunov function. Then, we establish the convergence of the tracking error to the target region $D_{e}$ defined in (11), on the basis of the Barbalat's Lemma. The validity of the procedure can be stated using the theory developed and applied in $[18,19,21,22]$.

The closed-loop dynamics is given by (32), and (38). We define the following truncated quadratic form, on the basis of the truncation presented in [19]:

$$
\begin{gathered}
\bar{V}_{s}= \begin{cases}V_{s} & \text { if } V_{s} \geq C_{b v s}, \\
C_{b v s} & \text { if } V_{s}<C_{b v s},\end{cases} \\
V_{s} \triangleq\left(\frac{1}{2}\right) s^{2},
\end{gathered}
$$

where $C_{b v s}$ is defined in (23). The form $\overline{V_{s}}$ has the following properties:

$$
\begin{array}{cc}
-V_{s}<-\overline{V_{s}}+C_{b v s}<0 & \text { if } V_{s}>C_{b v s}, \\
-V_{s}<-\overline{V_{s}}+C_{b v s}=0 & \text { if } V_{s}=C_{b v s}, \\
-V_{s} \leq 0=-\overline{V_{s}}+C_{b v s}=0 & \text { if } V_{s}<C_{b v s .} .
\end{array}
$$

The states of the closed-loop system may be grouped in the following vector:

$$
\bar{x}=\left[s, \tilde{c}, \tilde{C}_{d}^{\top}, \tilde{\theta}^{\top}\right]^{\top},
$$

where $\tilde{c}, \widetilde{C}_{d}$, and $\tilde{\theta}$ are defined in (26), (37), (27). We define the following function:

$$
\begin{gathered}
V(\bar{x}(t))=\overline{V_{s}}+V_{\theta}+V_{c}+V_{d}, \\
V_{\theta}=\left(\frac{1}{2}\right) \tilde{\theta}^{\top} \Gamma^{-1} \tilde{\theta}, \quad V_{c}=\left(\frac{1}{2}\right) \gamma_{c}^{-1} \widetilde{c}^{2}, \\
V_{d}=\left(\frac{1}{2}\right) \tilde{C}_{d}^{\top} \Gamma_{d}^{-1} \widetilde{C}_{d} .
\end{gathered}
$$

Notice that $V(\bar{x})$ does not satisfy standard conditions of Lyapunov theory for time-variable systems, shown in [18]. It satisfies the conditions of [19] instead, which we use to prove the boundedness of the closed-loop signals and the convergence of the tracking error to the residual set $D_{e}(11)$. The time derivative of $V_{s}$ along the trajectory (32) is

$$
\begin{aligned}
\dot{V}_{s}= & s \dot{s}=-a_{m} s^{2}-|b| \hat{c}^{2} s^{4}-|b|\left(\varphi^{\top} \hat{\theta}\right)^{2} s^{2} \\
& +(-1)|b| \hat{c}_{1}^{2} f_{1}^{2} s^{2}+\cdots+(-1)|b| \hat{c}_{l}^{2} f_{l}^{2} s^{2} \\
& +s\left(d-\varphi^{\top} \theta^{*}\right) \leq-a_{m} \delta^{2}-\tilde{c} \xi^{2}+\varsigma \varphi^{\top} \tilde{\theta} \\
& +(-1)|s| \tilde{C}_{d}^{\top} f \quad \text { if } V_{s} \geq C_{b v s},
\end{aligned}
$$

where the last inequality is obtained from (36). The time derivative of $\overline{V_{s}}$ can be derived from (39) and expressed on the basis of the above equation:

$$
\begin{aligned}
& \dot{\bar{V}_{s}} \\
& = \begin{cases}\dot{V}_{s} \leq-a_{m} s^{2}-\tilde{c} \xi^{2}+\delta \varphi^{\top} \tilde{\theta}+(-1)|\&| \tilde{C}_{d}^{\top} f & \text { if } V_{s} \geq C_{b v s}, \\
0 & \text { if } V_{s}<C_{b v s} .\end{cases}
\end{aligned}
$$

The time derivative $\dot{V}_{\theta}+\dot{V}_{c}+\dot{V}_{d}$ along trajectories (38) is

$$
\dot{V}_{\theta}+\dot{V}_{c}+\dot{V}_{d}= \begin{cases}(-1) \& \tilde{\theta}^{\top} \varphi+\tilde{c} \xi^{2}+\tilde{C}_{d} f|\delta| & \text { if } V_{s} \geq C_{b v s}, \\ 0 & \text { otherwise. }\end{cases}
$$

Therefore, $\dot{V}$ is given by

$$
\begin{aligned}
\dot{V} & =\dot{V}_{s}+\dot{V}_{\theta}+\dot{V}_{c}+\dot{V}_{d} \\
& = \begin{cases}\dot{V}_{s}+\dot{V}_{\theta}+\dot{V}_{c}+\dot{V}_{d} \leq-a_{m} s^{2} & \text { if } V_{s} \geq C_{b v s}, \\
0 & \text { if } V_{s}<C_{b v s} .\end{cases}
\end{aligned}
$$

The above equation can be rewritten in terms of $V_{s}$ defined in (40):

$$
\begin{gathered}
\dot{V} \leq-2 a_{m} V_{s} \quad \text { if } V_{s} \geq C_{b v s}, \\
\dot{V}=0 \quad \text { if } V_{s}<C_{b v s .}
\end{gathered}
$$

This implies that $\dot{V} \leq 0$ for all $t \geq t_{o}$. Thus, according to [19], all the closed-loop signals are bounded, that is, $(\delta, \widetilde{\theta}, \tilde{c}$, $\left.\widetilde{C}_{d}\right) \in L_{\infty}$, or equivalently, $\bar{x} \in L_{\infty}$, where $\bar{x}$ is defined in (42). As a consequence, $\left(V, \bar{V}_{s}, V_{s}, V_{\theta}, V_{c}, V_{d}\right) \in L_{\infty}$, according to the definitions in (39) and (43). The boundedness of $\bar{x}$ implies $\left(e, \ldots, e^{(n-1)}\right) \in L_{\infty}$ according to (12); hence, $\dot{s} \in$ $L_{\infty}$ [22], and $\dot{\bar{V}}_{s} \in L_{\infty}$. To establish the convergence of the tracking error, we begin by expressing the equation ahead in terms of $\overline{V_{s}}$, according to the definition of $\overline{V_{s}}$ in (39) and the properties of (41):

$$
\begin{aligned}
\dot{V} & \leq-2 a_{m} V_{s}<-2 a_{m} \overline{V_{s}}+2 a_{m} C_{b v s} \leq 0 \quad \text { if } V_{s} \geq C_{b v s}, \\
\dot{V} & =0=2 a_{m}\left(-\overline{V_{s}}+C_{b v s}\right)=0 \quad \text { if } V_{s}<C_{b v s}, \\
& \Longrightarrow \dot{V} \leq-2 a_{m} \overline{V_{s}}+2 a_{m} C_{b v s} \leq 0 .
\end{aligned}
$$

We reorganize the above equation and integrate as follows:

$$
2 a_{m} \int_{t_{o}}^{t}\left(\overline{V_{s}}-C_{b v s}\right) d \tau+V(t) \leq V\left(t_{o}\right) .
$$


Thus, $\left(\overline{V_{s}}-C_{b v s}\right) \in L_{1}$. Recall that $\left(\overline{V_{s}}, \dot{\overline{V_{s}}}\right) \in L_{\infty}$. Thus, $\left(\overline{V_{s}}-\right.$ $\left.C_{b v s}\right) \in L_{\infty} \cap L_{1},(d / d t)\left(\overline{V_{s}}-C_{b v s}\right) \in L_{\infty}$. By invoking the Barbalat Lemma [23], we obtain $\left(\overline{V_{s}}-C_{b v s}\right) \rightarrow 0$ as $t \rightarrow \infty$. In turn, this implies that $V_{s} \rightarrow D_{v s}$, being $D_{v s}$ defined as

$$
D_{v s}=\left\{V_{s}: V_{s} \leq C_{b v s}\right\}
$$

where $C_{b v s}$ is defined in (23). $\&$ can be expressed in terms of $V_{s}$, on the basis of the definition of $V_{s}$ in (40):

$$
s=\sqrt{2 V_{s}}
$$

In the following, we analyze the convergence of $§$. Taking into account the definition of $C_{b v s}$ in (23), the fact that $V_{s}$ converges to $D_{v s}$ defined in (51), and (52) the convergence of 8 is:

$$
\begin{gathered}
\lim _{t \rightarrow \infty} \S=D_{s}, \quad D_{s}=\left\{\&:|\delta| \leq C_{b s}\right\}, \\
C_{b s}=\sqrt{2 C_{b v s}}=\lambda^{n-1} C_{b e} .
\end{gathered}
$$

To analyze the convergence of $e$, we begin by expressing $e$ in terms of $\&$, according to (12):

$$
e=\frac{1}{(p+\lambda)^{n-1}} \S .
$$

The convergence of the tracking error may be established on the basis of (53) and (54) [18, 21]:

$$
\lim _{t \rightarrow \infty} e(t)=D_{e}, \quad D_{e}=\left\{e:|e| \leq C_{b e}\right\},
$$

which means that the tracking error $e$ converges to a residual set whose size is of the user's choice.

\section{Application to a Permanent Magnet Synchronous Motor}

A PMSM is a kind of highly efficient and high-powered motor. The benefits of the PMSM are discussed in [24, 25]. The PMSM has the following difficulties [26]: (i) it is highly nonlinear; (ii) the parameters of the physical model experience unknown time-varying behavior, for example, the stator resistance $R$ and the friction coefficient $B$; (iii) unknown external disturbances appear, that is, the load torque disturbance $T_{L}$. Moreover, by varying the permanent magnet flux $\lambda_{a f}$ the state $\omega$ exhibits a pitchfork bifurcation [27]. All the mentioned facts lead to response deterioration of many controllers, specially for high-speed and highprecision tasks in real applications [28].

In view of the complex behavior, with parameters varying with respect to time and state plane, the scheme developed in this work is suitable. We apply the developed scheme by simulation, to a PMSM whose model and parameters are presented in [29]. Therein, the possible manipulated inputs are $u_{d}$ and $u_{q}$; and $i_{d}, i_{q}$, and $\omega$ are possible outputs. We choose the motor angular frequency $\omega$ as the output to be controlled and the $d$-axis voltage $u_{d}$ as the control input, that is, $y=\omega, u=u_{d}, v_{d}=0$. A similar choice is made in [24], where an adaptive controller is derived for a PMSM. After performing a step response analysis, the variable $\omega$ exhibits a second-order behavior when a step change is introduced in $u_{d}$. This second order behavior was already noticed in [29]. Thus, we use the regression model (6) with $n=2$. We can summarize the basic features of the plant as

$$
\begin{gathered}
\quad n=2, \quad \operatorname{sgn}(b)=+1, \\
l=3, \quad f_{1}=|\dot{y}|, \quad f_{2}=|y|, \\
f_{3}=1, \quad \Longrightarrow f=[|\dot{y}|,|\mathrm{y}|, 1]^{\top}, \\
\phi=\left[y^{2}, y^{3}\right]^{\top}, \quad \varphi_{a}=-\ddot{y}_{d}+\lambda_{o} \dot{e}, \\
\Longrightarrow \varphi=\left[-\phi^{\top},-\varphi_{a}-a_{m} \&\right]^{\top} .
\end{gathered}
$$

We used a factorization with terms $y^{2}$ and $y^{3}$ to take into account the presence of the pitchfork bifurcation. According to [30] the normal form of the pitchfork bifurcation gives a description of the system behavior in a tight neighborhood of the bifurcation point. For the case of the pitchfork bifurcation, the normal form includes the terms $y^{2}$ and $y^{3}$. Although the system usually works in different regions far from the bifurcation point, we wanted to include the behavior in the neighborhood of the bifurcation point. Thus, we included the terms of the normal form for the pitchfork bifurcation [30], that is, $y^{2}$ and $y^{3}$. The terms 1 and $y$ are not included in $\phi$ because they are already present in $d$. The remaining parameters of the controller are defined on the basis of the parameters in (56):

$$
\begin{aligned}
& e=y-y_{d}, \quad \delta=(p+\lambda) e=\dot{e}+\lambda e \Longrightarrow \lambda_{o}=\lambda, \\
& C_{b v s}=\left(\frac{1}{2}\right)\left(\lambda C_{b e}\right)^{2} \text {, } \\
& u=(-1) \operatorname{sgn}(b) \hat{c}^{2} \delta^{3}+(-1) \operatorname{sgn}(b)(\varphi \hat{\theta})^{2} \& \\
& +(-1) \operatorname{sgn}(b) \&\left(\hat{c}_{1}^{2} f_{1}^{2}+\hat{c}_{2}^{2} f_{2}^{2}+\hat{c}_{3}^{2} f_{3}^{2}\right) \\
& =(-1) \operatorname{sgn}(b) \&\left(\hat{c}^{2} \delta^{2}+(\varphi \hat{\theta})^{2}+\hat{c}_{1}^{2} f_{1}^{2}+\hat{c}_{2}^{2} f_{2}^{2}+\hat{c}_{3}^{2} f_{3}^{2}\right) \text {. }
\end{aligned}
$$

In addition, we choose:

$$
\begin{gathered}
\lambda=3, \quad a_{m}=10, \quad a_{m, 1}=70, \\
a_{m, o}=1225 \text { (for the reference model) } \quad C_{b e}=0.1, \\
\gamma_{c}=20, \quad \Gamma_{d}=\operatorname{diag}[20,20,20], \\
\Gamma=\operatorname{diag}[0.4,0.4,0.003] .
\end{gathered}
$$

The results are shown in Figure 1. The simulation shows expected results: all the closed-loop signals remain bounded, and transient values of the tracking error remain in a small interval. It is worthy of note that the transient values of $e$ depend on its initial value and the initial values of the adjustment errors. To show the effect of the time-varying behavior of the model coefficients, we change the stator resistance $R$ from 1.4 to $1.7 \Omega$ at the time instant $0.6 \mathrm{sec}$. Results are shown in Figure 2.

In addition, we consider the variation of the damping constant $B$ from 0.00038818 to $0.00046582 \mathrm{Nm} /(\mathrm{rad} / \mathrm{s})$ at the 


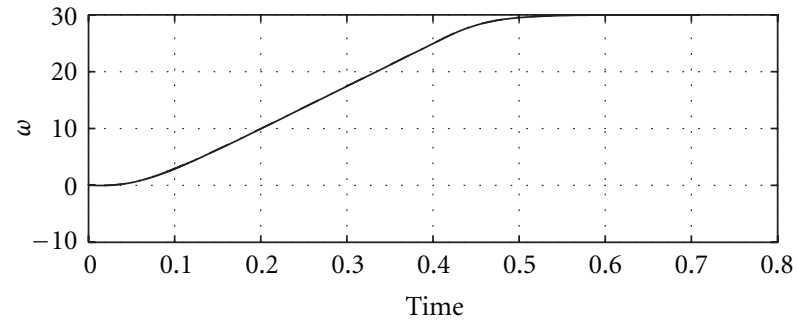

- Rotor speed

...- Reference

(a)

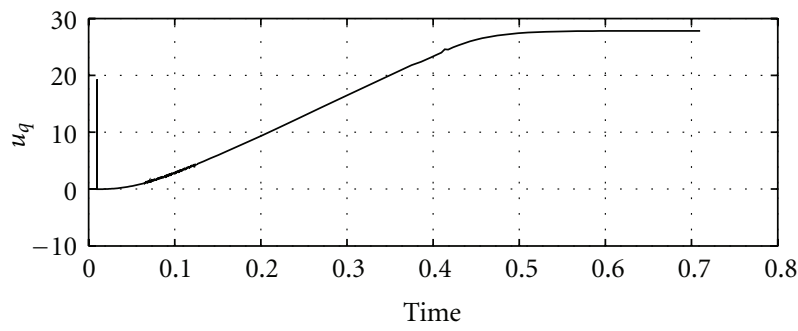

(b)

FIGURE 1: Transient behavior of the output and control input.

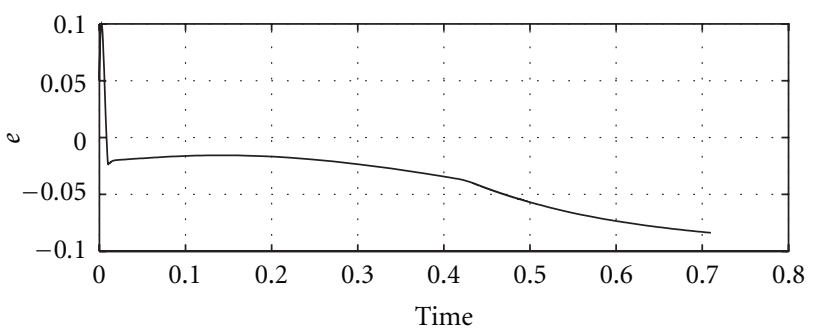

(a)

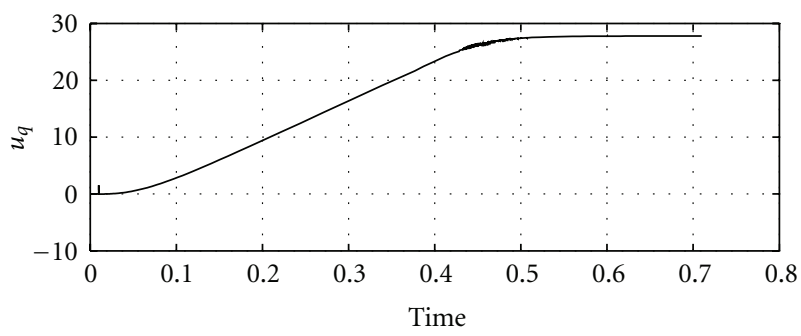

(b)

FIGURE 2: Performance of the tracking error and control input varying the resistance value.

instant $0.6 \mathrm{sec}$. Results are shown in Figure 3. Notice that in the three cases the final value of $|e|$ is less than $C_{b e}=$ 0.1 . Moreover, the control input $u$ belongs to the interval [-200 200] V. Notice in Figures 2 and 3 that the effect of the disturbance on the tracking error is almost negligible. Nevertheless, the control input experiences a large variation, as can be seen in Figure 3 .

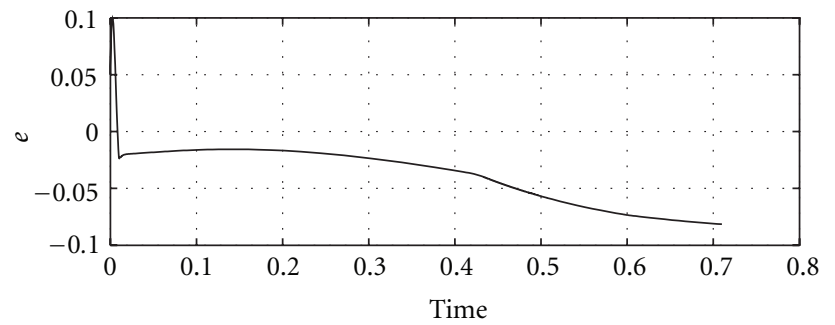

(a)

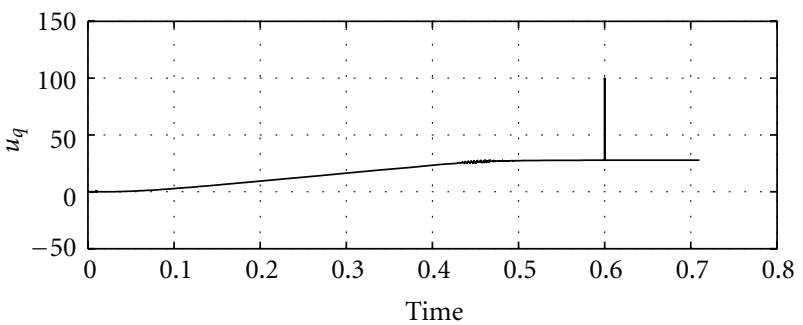

(b)

Figure 3: Performance of the tracking error and control input varying the damping constant value.

\section{Conclusions}

In this work, we have proposed a control scheme for highly nonlinear plants, based on a simple plant model with polynomial approximators, which provides an adequate description of transient behavior. It is worth noticing the fact that benefits Ri to Rviii (Section 2) are achieved at the same time, with minimal requirements on the knowledge of the plant. Indeed, the relative degree and the signum of the control gain can be established by a previous step response analysis. Many techniques are combined at the same time: sliding surface MRAC, dead zone-type update law, robustifying auxiliary control, approximation techniques, and truncation of the quadratic forms.

The disadvantage of polynomials as approximators is that they may be less accurate than other techniques, for example, neural networks or fuzzy sets, leading to higher approximation error. Since the approximation error is bounded, it can be handled by means of robustness techniques without requiring the upper bound to be known. Moreover, we considered the coefficients of the terms $y, \ldots, y^{(n-1)}$ as timevarying but bounded, with constant and unknown bounds. Then, we handled this by means of robust control, without requiring the upper bounds to be known.

We handled the time-varying behavior of the control gain by means of robustness techniques, without using the Nussbaum gain method. The redefinition of the plant terms in terms of adjustment errors and adjustment parameters is a fundamental step. The resulting expression allows a straightforward definition of the control law. The variation of the control gain implies that the terms involving adjusted parameters cannot be cancelled. Rather, we attenuate its effect by means of squared terms and handle the residual error by means of an additional control term that is only a function of the sliding surface. The resulting expression 
for $V, \dot{V}$, and the design is simpler in comparison with the Nussbaum technique.

\section{Appendix}

\section{Proof of $(34)$}

As the first step, we factorize several summands of (34) and apply the property (8):

$$
\begin{aligned}
-|b| \hat{c}^{2} \delta^{4} & +\hat{c} \delta^{2} \leq-b_{m} \hat{c}^{2} \delta^{4}+\hat{c} \delta^{2} \\
& =-\left[\sqrt{b_{m}} \hat{c} \delta^{2}-\frac{1}{2 \sqrt{b_{m}}}\right]^{2}+\left(\frac{1}{2 \sqrt{b_{m}}}\right)^{2} \leq\left(\frac{1}{2 \sqrt{b_{m}}}\right)^{2} .
\end{aligned}
$$

Likewise, we obtain:

$$
\begin{gathered}
(-1)|b|\left(\varphi^{\top} \hat{\theta}\right)^{2} \S^{2}-\S \varphi^{\top} \hat{\theta} \leq\left(\frac{1}{2 \sqrt{b_{m}}}\right)^{2}, \\
(-1)|b| \hat{c}_{1}^{2} f_{1}^{2} \S^{2}+\hat{c}_{1}|\&| f_{1} \leq\left(\frac{1}{2 \sqrt{b_{m}}}\right)^{2}, \\
\vdots \\
(-1)|b| \hat{c}_{l}^{2} f_{l}^{2} \S^{2}+\hat{c}_{l}|\&| f_{l} \leq\left(\frac{1}{2 \sqrt{b_{m}}}\right)^{2} .
\end{gathered}
$$

By adding (A.1) and (A.2) we obtain:

$$
\begin{aligned}
& -|b| \hat{c}^{2} \delta^{4}+(-1)|b|\left(\varphi^{\top} \hat{\theta}\right)^{2} \delta^{2}+(-1)|b| \hat{c}_{1}^{2} f_{1}^{2} \delta^{2} \\
& +\cdots+(-1)|b| \hat{c}_{l}^{2} f_{l}^{2} \delta^{2}+\hat{c} \delta^{2}-8 \varphi^{\top} \hat{\theta}+\hat{c}_{1}|\&| f_{1} \\
& +\cdots+\hat{c}_{l}|\&| f_{l} \leq(2+l)\left(\frac{1}{2 \sqrt{b_{m}}}\right)^{2} .
\end{aligned}
$$

As a second step, we use the definition of $c^{*}$ in (22) to rewrite the term $-c^{*} \delta^{2}$ :

$$
\begin{aligned}
-c^{*} f^{2} & =-(2+l) \frac{\delta^{2}}{2 C_{b v s}}\left(\frac{1}{2 \sqrt{b_{m}}}\right)^{2} \\
& \leq-(2+l)\left(\frac{1}{2 \sqrt{b_{m}}}\right)^{2} \quad \text { if } s^{2} \geq 2 C_{b v s} .
\end{aligned}
$$

As the third step, we add (A.3) and (A.4):

$$
\begin{aligned}
& -|b| \hat{c}^{2} \delta^{4}+(-1)|b|\left(\varphi^{\top} \hat{\theta}\right)^{2} \delta^{2}+(-1)|b| \hat{c}_{1}^{2} f_{1}^{2} 8^{2} \\
& +\cdots+(-1)|b| \hat{c}_{l}^{2} f_{l}^{2} \delta^{2}+\hat{c} \delta^{2}-\diamond \varphi^{\top} \hat{\theta}+\hat{c}_{1}|\&| f_{1} \\
& +\cdots+\widehat{c}_{l}|\&| f_{l}-c^{*} \delta^{2} \leq 0 \text { if } \AA^{2} \geq 2 C_{b v s} \text {, }
\end{aligned}
$$

which is (34).

\section{Acknowledgments}

This work was partially supported by Universidad Nacional de Colombia-Manizales, project 12475, Vicerrectoría de Investigación, DIMA, resolution number VR-2185.

\section{References}

[1] K. S. Narendra and K. George, "Adaptive control of simple nonlinear systems using multiple models," in Proceedings of the American Control Conference (ACC '02), pp. 1779-1784, Anchorage, AK, USA, May 2002.

[2] I. Kanellakopoulos, P. V. Kokotovic, and A. S. Morse, "Systematic design of adaptive controllers for feedback linearizable systems," IEEE Transactions on Automatic Control, vol. 36, no. 11, pp. 1241-1253, 1991.

[3] J. Nakanishi, J. A. Farrell, and S. Schaal, "Composite adaptive control with locally weighted statistical learning," Neural Networks, vol. 18, no. 1, pp. 71-90, 2005.

[4] H. A. Yousef and M. A. Wahba, "Adaptive fuzzy mimo control of induction motors," Expert Systems with Applications, vol. 36, no. 3, pp. 4171-4175, 2009.

[5] A. C. Huang and Y. S. Kuo, "Sliding control of non-linear systems containing time-varying uncertainties with unknown bounds," International Journal of Control, vol. 74, no. 3, pp. 252-264, 2001.

[6] A. C. Huang and Y. C. Chen, "Adaptive multiple-surface sliding control for non-autonomous systems with mismatched uncertainties," Automatica, vol. 40, no. 11, pp. 1939-1945, 2004.

[7] C. H. Chen, C. M. Lin, and T. Y. Chen, "Intelligent adaptive control for MIMO uncertain nonlinear systems," Expert Systems with Applications, vol. 35, no. 3, pp. 865-877, 2008.

[8] Y. Feng, C. Y. Su, H. Hong, and S. S. Ge, "Robust adaptive control for a class of nonlinear systems with generalized PrandtlIshlinskii hysteresis," in Proceedings of the 46th IEEE Conference on Decision and Control 2007 (CDC'07), pp. 4833-4838, New Orleans, Calif, USA, December 2007.

[9] K. S. Narendra and J. Balakrishnan, "Adaptive control using multiple models," IEEE Transactions on Automatic Control, vol. 42, no. 2, pp. 171-187, 1997.

[10] S. S. Ge and J. Wang, "Robust adaptive tracking for timevarying uncertain nonlinear systems with unknown control coefficients," IEEE Transactions on Automatic Control, vol. 48, no. 8, pp. 1463-1469, 2003.

[11] C. P. Bechlioulis and G. A. Rovithakis, "Adaptive control with guaranteed transient and steady state tracking error bounds for strict feedback systems," Automatica, vol. 45, no. 2, pp. 532-538, 2009.

[12] C. Y. Su, Y. Feng, H. Hong, and X. Chen, "Adaptive control of system involving complex hysteretic nonlinearities: a generalised Prandtl-Ishlinskii modelling approach," International Journal of Control, vol. 82, no. 10, pp. 1786-1793, 2009.

[13] Y. Feng, C. Y. Su, and H. Hong, "Universal construction of robust adaptive control laws for a class of nonlinear systems preceded by generalized Prandtl-Ishlinskii representation," in Proceedings of the 3rd IEEE Conference on Industrial Electronics and Applications (ICIEA '08), pp. 153-158, Singapore, Singapore, June 2008.

[14] C. F. Hsu, C. M. Lin, and T. T. Lee, "Wavelet adaptive backstepping control for a class of nonlinear systems," IEEE Transactions on Neural Networks, vol. 17, no. 5, pp. 1175-1183, 2006.

[15] C. Y. Lee, "Adaptive control of a class of nonlinear systems using multiple parameter models," International Journal of Control, Automation and Systems, vol. 4, no. 4, pp. 428-437, 2006.

[16] S. Labiod and T. M. Guerra, "Adaptive fuzzy control of a class of SISO nonaffine nonlinear systems," Fuzzy Sets and Systems, vol. 158, no. 10, pp. 1126-1137, 2007. 
[17] J. Spooner, M. Maggiore, R. Ordóñez, and K. Passino, Stable Adaptive Control and Estimation for Nonlinear Systems: Neural and Fuzzy Approximator Techniques, John Wiley \& Sons, New York, NY, USA, 2002.

[18] J. J. Slotine and W. Li, Applied Nonlinear Control, Prentice Hall, Englewood Cliffs, NJ, USA, 1991.

[19] B. B. Peterson and K. Narendra, "Bounded error adaptive control," IEEE Transactions on Automatic Control, vol. AC-27, no. 6, pp. 1161-1168, 1982.

[20] K. Astrom and B. Wittenmark, Adaptive Control, AddisonWesley Publishing Company Inc, Reading, Mass, USA, 1995.

[21] X. S. Wang, C. Y. Su, and H. Hong, "Robust adaptive control of a class of nonlinear systems with unknown dead-zone," Automatica, vol. 40, no. 3, pp. 407-413, 2004.

[22] J. Wu, D. Pu, and H. Ding, "Adaptive robust motion control of SISO nonlinear systems with implementation on linear motors," Mechatronics, vol. 17, no. 4-5, pp. 263-270, 2007.

[23] P. A. Ioannou and J. Sun, Robust Adaptive Control, PrenticeHall PTR, Upper Saddle River, NJ, USA, 1996.

[24] J. Zhou and Y. Wang, "Real-time nonlinear adaptive backstepping speed control for a PM synchronous motor," Control Engineering Practice, vol. 13, no. 10, pp. 1259-1269, 2005.

[25] L. Liu, W. Liu, and D. A. Cartes, "Particle swarm optimizationbased parameter identification applied to permanent magnet synchronous motors," Engineering Applications of Artificial Intelligence, vol. 21, no. 7, pp. 1092-1100, 2008.

[26] F. Morel, X. Lin-Shi, J. M. Rétif, and B. Allard, "A predictive current control applied to a permanent magnet synchronous machine, comparison with a classical direct torque control," Electric Power Systems Research, vol. 78, no. 8, pp. 1437-1447, 2008.

[27] Z. Jing, C. Yu, and G. Chen, "Complex dynamics in a permanent-magnet synchronous motor model," Chaos, Solitons and Fractals, vol. 22, no. 4, pp. 831-848, 2004.

[28] A. M. Harb, "Nonlinear chaos control in a permanent magnet reluctance machine," Chaos, Solitons and Fractals, vol. 19, no. 5, pp. 1217-1224, 2004.

[29] P. Pillay and R. Krishnan, "Control characteristics and speed controller design for a high performance permanent magnet synchronous motor drive," IEEE Transactions on Power Electronics, vol. 5, no. 2, pp. 151-159, 1989.

[30] Y. A. Kuznetsov, Elements of Applied Bifurcation Theory, Springer-Verlag, New York, NY, USA, 3rd edition, 2004. 

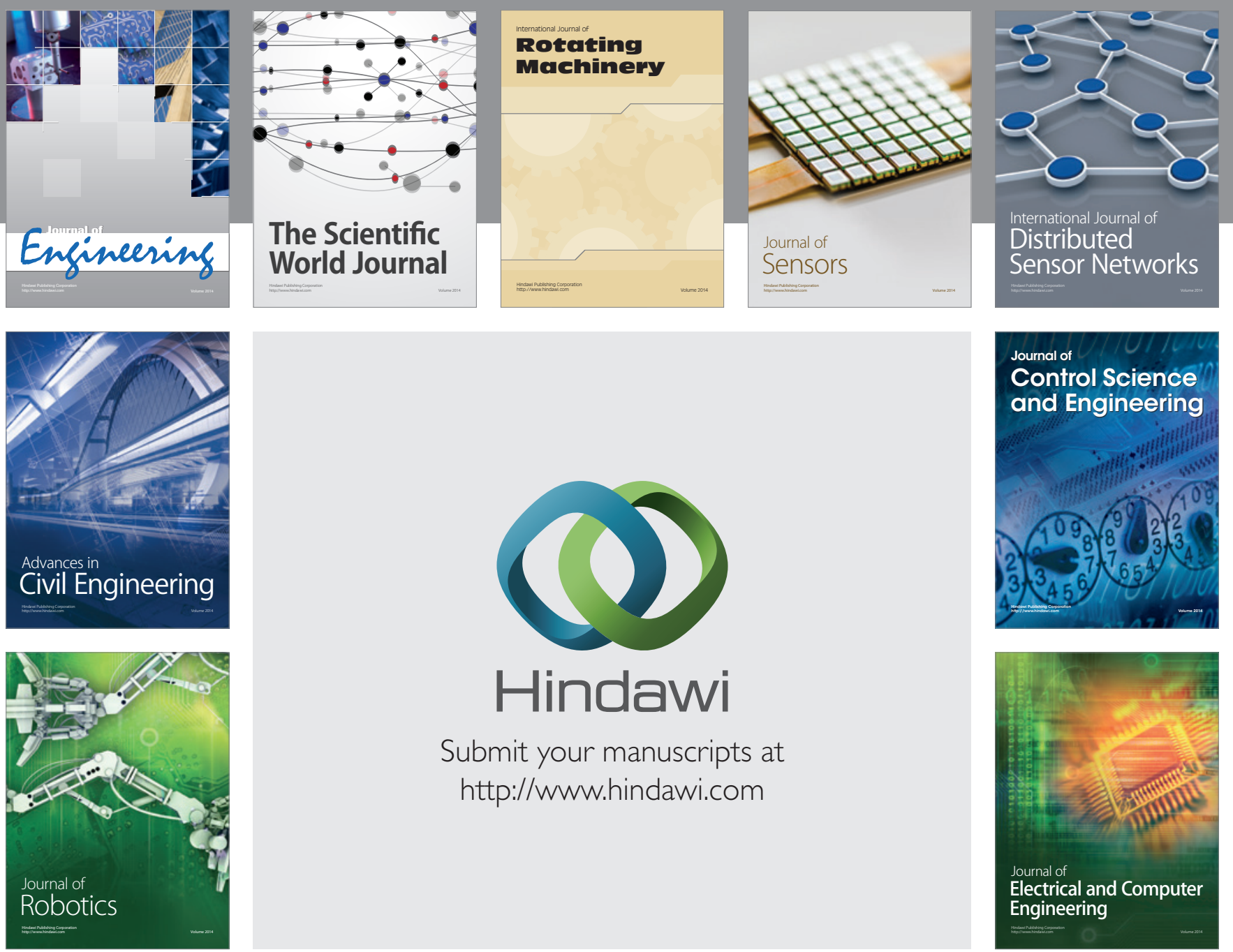

Submit your manuscripts at

http://www.hindawi.com
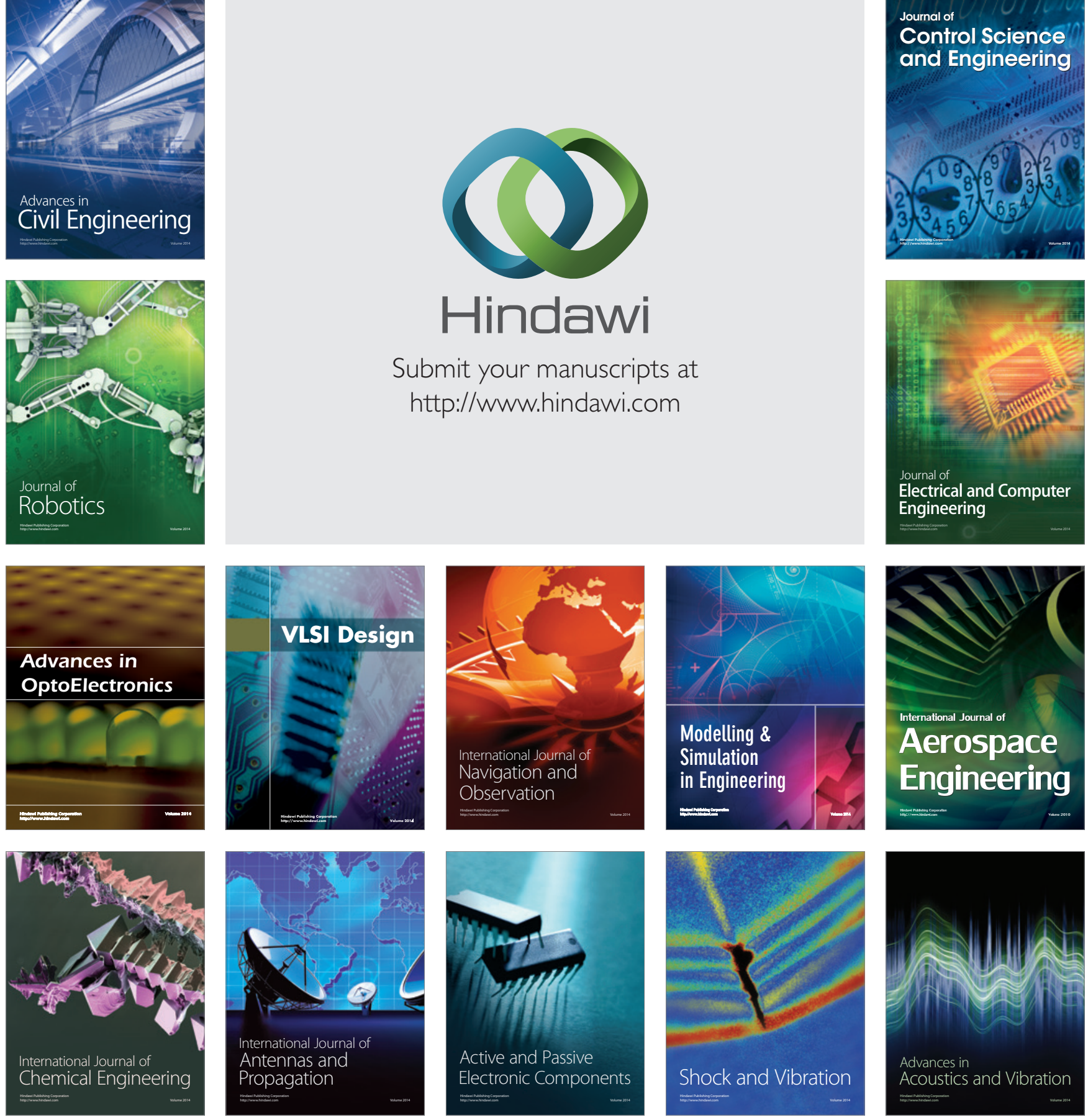